\title{
Implementing Spontaneous Breathing Trials - But a Piece of the Puzzle
}

Both adult and pediatric ICUs have adopted respiratory therapist- or nurse-driven protocols that help advance the care of patients and may improve outcomes. In this issue of Respiratory CARE, Krawiec and colleagues ${ }^{1}$ describe the creation of a spontaneous breathing trial (SBT) protocol and some challenges of early implementation, especially that of consistent screening. This quality improvement project was performed in a mixed medical-surgical pediatric ICU population (with the exception of postoperative cardiac patients) with the goal of performing twice-daily SBTs in the identified candidates. The use of SBTs in the pediatric ICU is but one small component of what comprehensive protocolized ventilator weaning could look like. The motivation to develop an evidence-based ventilator weaning protocol to help separate patients from the ventilator when the underlying reason for intubation has been sufficiently, but not completely, reversed is warranted due to the growing list of complications associated with invasive ventilation. For the majority of intubated patients, a standardized protocol could balance the risks of premature extubation with the risks of a protracted ventilator course.

The process of weaning the ventilator is more than performing an SBT, although the term has become synonymous with performing a trial of extubation readiness. Perhaps this is because weaning does not have an accepted definition. This ambiguity introduces significant challenges for investigating ventilator weaning, because we cannot yet begin to describe even the most basic disease-specific characteristics, such as the median duration that patients with more versus less severe lung disease spend in the weaning process. If one considers the process of ventilator weaning to encapsulate the time between maximal ventilator support to the removal of the endotracheal tube, then it becomes apparent that there are more questions than answers with regard to ventilator discontinuation.

Let's consider a hypothetical patient's course on the ventilator (Fig. 1). The patient is intubated and ventilator

\footnotetext{
Mr DiBlasi discloses relationships with Draeger, Mallinckrodt, and Aerogen. Dr Hartmann has no conflicts to disclose.
}

Correspondence: Dr Silvia Hartmann, 4800 Sand Point Way NE, M/S FA2.112, Seattle, WA 98105. silvia.hartmann@ seattlechildrens.org.

DOI: $10.4187 /$ respcare. 05826 settings are escalated to achieve oxygenation and ventilation goals. Typically the patient spends a period of time at maximal or near-maximal ventilator settings, which can be visualized as a plateau phase. When the team feels that the

See the Original Study on Page 1233

patient has demonstrated stability and the process that led to the need for invasive mechanical ventilation is sufficiently reversed, then the ventilator settings are decreased, often in a step-wise manner, until the patient meets screening criteria for the performance of an SBT. When an SBT is performed, the patient's vital signs, end-tidal carbon dioxide readings, tidal volume, and sometimes blood gases are compared to predetermined criteria for failure of the test. If the patient passes an SBT, the decision will be made whether to remove the endotracheal tube. If the decision is an affirmative one, then he or she will be extubated when possible. Some patients experience a significant delay of several hours between SBT success and extubation. ${ }^{2}$

There is a general lack of clinical investigation around ventilator de-escalation compared to escalation, which leaves weaning to be something of an art rather than a science. Some questions for thought follow.

\section{How Long Should Patients With Respiratory Failure Be in the Plateau Phase of Ventilator Support Before Trials of Decreased Settings Are Attempted?}

It is unknown how long patients with variable severity of lung disease need to spend at maximal or near-maximal ventilator support. Intuitively, it would make sense that as soon as gas exchange is stabilized, lungs are recruited near functional residual capacity (FRC), and the patient has adequate respiratory drive, then ventilator settings should be decreased. Recruited lung usually requires less pressure to remain open than it took for the process of recruitment. However, the transpulmonary pressure required by the patient to maintain adequate lung inflation depends on FRC and lung mechanics measurements. We may make guesses that patients are near FRC on the basis of chest radiographs, but currently there is a lack of simple objective tools to reliably measure FRC at the bedside. Electrical 


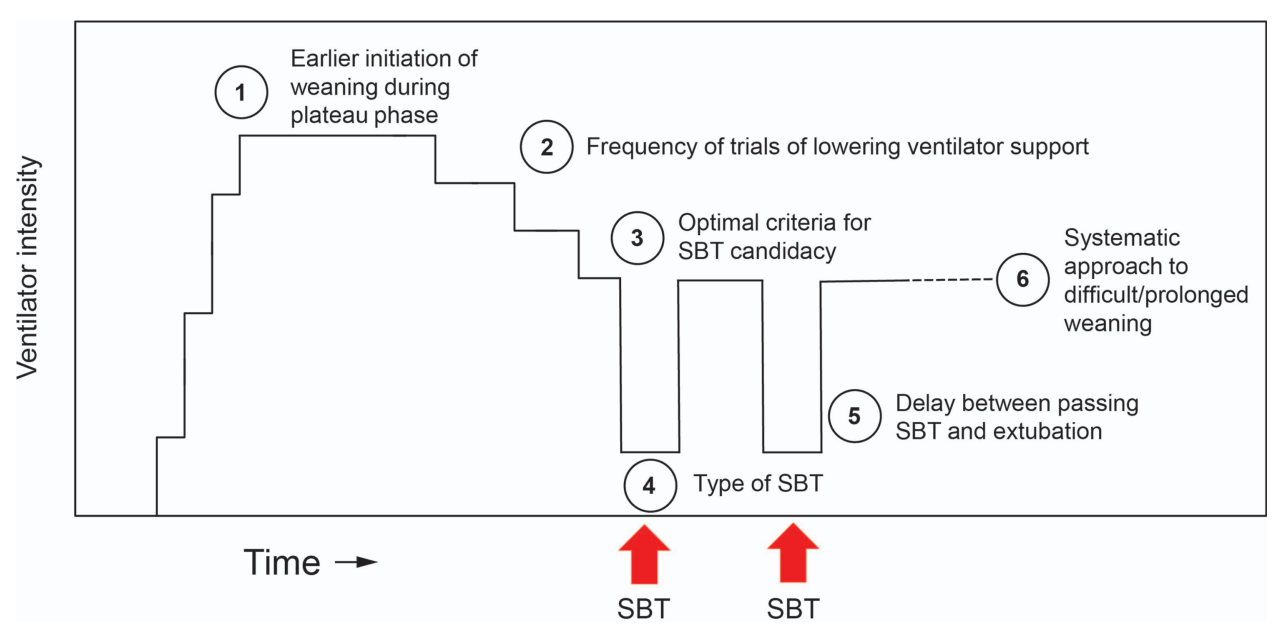

Fig. 1. Areas of inquiry around weaning from mechanical ventilation. SBT = spontaneous breathing trial.

impedance tomography, volumetric carbon dioxide measurements, and esophageal manometry may help bridge this need in the future, but none of these measurements are currently widely available in the pediatric population.

\section{How Frequently Can the Ventilator Settings Be Decreased With the Workflow in the ICU?}

Sequential decreases in ventilator settings until a patient meets criteria for an SBT generally occur in a haphazard way. It typically occurs based on the schedule of health care providers rather than patient tolerance. Due to the lack of definitive data related to weaning, magnitude and frequency of ventilator setting reduction in pediatric ICU may be driven largely by anecdote, conventional wisdom, or clinician availability. The increased use of volume-targeted ventilator modes in the pediatric ICU that sequentially adjust peak inspiratory pressure in response to improvement in lung mechanics may help bridge part of this delay.

Most studies of protocolized ventilator weaning refer solely to the use of screening criteria for SBT and SBT performance, similar to the investigation by Krawiec et al. ${ }^{1}$ Based on the expanded definition of ventilator weaning proposed above, these types of protocols miss the opportunity to standardize and schedule trials of lower settings throughout each day that a patient spends connected to a ventilator. From the adult literature, it does appear that the more frequently that a patient can be assessed for decreased settings, the more successful the process is at decreasing duration of mechanical ventilation. ${ }^{3}$ However, ventilator modes or software that can assess the patient for decreased support on the order of minutes may be the answer to this question. The current investigation of such software for pediatric patients can only be implemented in larger children, which misses a significant number of in- tubated patients and has only been studied during the end of the ventilator course, when settings were reasonably low. ${ }^{4}$ Additionally, it is unclear whether the average patient with respiratory failure spends more time in the plateau phase or more time receiving sequential decreases in ventilator settings before meeting SBT screening criteria. Knowing this would help create a target for protocolization that would have greater impact on ventilator duration.

\section{Are We Using the Right Screening Criteria for the Performance of an SBT? Are They Too Conservative, and Do They Wait Too Long for Resolution of Lung Disease?}

The use of an SBT to detect readiness for endotracheal extubation at low-moderate ventilator settings compared to gradual weaning and extubation has good evidence for reducing time on the ventilator in adults and pediatrics. ${ }^{5,6}$ However, there have been no studies comparing different screening criteria with time to SBT performance and subsequent extubation failure rate. Many studies and institutions use a list of variables meant to assure that patients have near-normal oxygenation, ventilation, cardiovascular capacity, and neurologic status. Recently the RESTORE trial $^{7}$ of protocolized sedation used an oxygenation index (OI) or oxygenation saturation index (OSI) of 6 or less to replace the oxygenation and ventilation criteria for patients with acute respiratory failure. We hypothesize that these criteria may lead to earlier SBT performance because more subjects failed an initial SBT compared to a previous study of pediatric ICU subjects using more traditional screening criteria. ${ }^{2,8}$ This is solely a hypothesis as the groups are not directly comparable; one group was composed only of subjects with lung disease, and the other was heterogeneous, including a notable minority of subjects with neurologic disorders. This is an important dis- 
tinction because a review of ventilator weaning protocols in adults indicates that patients with neurologic impairment as the underlying reason for mechanical ventilation do not benefit from traditional ventilator weaning protocols. ${ }^{9}$ No study to date has compared the timing of SBT performance using different screening criteria, such as OI/OSI versus a traditional list of parameters.

\section{What is the Best Way to Perform an SBT for the Average Pediatric ICU Patient? How Does That Differ from the Pediatric Patient Who is at High Risk of Extubation Failure?}

SBT and extubation readiness trial (ERT) have been used interchangeably by many, as used in the preceding discussion. A distinction ought to be made between the two. An SBT should inform the clinician as to whether a patient can initiate breaths on the ventilator, regardless of the level of support received. This is the first step in a process where the patient may be ready to control his or her own minute ventilation because it establishes adequacy of respiratory drive. On the other hand, an ERT should require that a patient pass an SBT and in addition tolerate minimal ventilator settings. The idea is to choose settings that best mimic the conditions a patient would experience once extubated, with the caveat that work imposed by upper airway resistance-normal and abnormal-cannot be evaluated. ERTs are performed in a variety of ways that many believe fulfill this idea. An ERT can be performed in a pressure support mode of ventilation, with CPAP alone, or without the ventilator using a T-piece. Using some amount of pressure support in addition to a low level of PEEP is the most common ERT used in the pediatric ICU. ${ }^{10}$ Many centers use increasing levels of pressure support, adapted from a trial of ventilator weaning, to compensate for theoretical increases in airway resistance with smaller diameter endotracheal tubes. ${ }^{11}$ Other centers use a fixed low level of pressure support for all tube sizes, on the basis of studies that show that work of breathing is higher after extubation compared to on the ventilator with pressure support or CPAP. ${ }^{12,13}$

The endotracheal tube does not impose significant additional airway resistance at the physiologic flows generated by pediatric patients, as smaller patients demand an overall lower flow through their smaller tubes compared to larger patients. The use of scaled pressure support levels based on tube size during an ERT is more likely to mask respiratory insufficiency in some specific groups of patients compared to the other methods of performing an ERT. The characteristics of the group of patients who could pass an ERT with scaled pressure support but would fail an ERT done with low levels of pressure support or CPAP is unknown, including the likelihood of needing respiratory support after extubation. This group of patients may have poor pulmonary reserve and have a higher risk of re-intubation or need higher levels of noninvasive positive pressure support. Although there is no specific evidence, we hypothesize that most children who pass an ERT that tests children using higher levels of pressure support likely have good pulmonary reserve and will do well after extubation. For the average child in the pediatric ICU, the type of ERT probably does not have a clinical effect, but for a child at higher risk of a failed extubation, such as one with neuromuscular weakness, an ERT with low or no pressure support or even a T-piece may be more appropriate to avoid re-intubation.

\section{How Can We Minimize the Delay Between Passing an SBT and Removal of the Endotracheal Tube From the Patient's Trachea?}

Endotracheal extubation certainly is not as urgent as endotracheal intubation can be, but it should not be significantly delayed after an SBT is passed. Ferguson et $\mathrm{al}^{2}$ noted a median delay of $5.5 \mathrm{~h}$ and an upper range of $26 \mathrm{~d}$ between passing an SBT and endotracheal extubation. The most common reason reported was excessive sedation in approximately $45 \%$ of subjects, followed by physician preference in $17.5 \% .^{2}$ Certainly the coordination of sedation administration and extubation is an obvious target for intervention to minimize delayed extubation. Physician preference may include such challenges as available staffing to observe patients after extubation; this could be resolved by different staffing models. The reasons for delayed extubation after passing an SBT remain partially explored.

\section{Is There a Better Way to Implement Systematic Assessment of Patients Who Fail SBTs?}

When a patient fails an SBT, the health care providers should assess a list of possible causes and address those that are amenable to intervention. Examples include administration of additional diuretic therapy to improve lung and chest wall compliance, repletion of electrolyte deficiencies, physical therapy for deconditioning, and performance of indirect calorimetry to exclude overfeeding and excessive carbon dioxide production. The list is long enough that it is a challenge to recite all elements from memory. Checklists and decision support tools in the electronic medical record have become a popular method to help clinicians consistently consider multiple issues in specific patient populations and could be applied to the cohort that has undergone unsuccessful testing for extubation readiness.

Weaning should be a process that centers on repeated challenges to the patient to accept an increased amount of the work of breathing that is being shared with the venti- 


\section{EDITORIALS}

lator. Steps in this process should focus on patient readiness rather than health care provider convenience, such as making ventilator changes on rounds rather than multiple times a day. We hope that the answers to the above questions, as well as the many unasked questions, help those who care for ventilated children know what to do to separate the child from the ventilator and studies of implementation help us know how it can actually be done.

Silvia M Hartmann MD

Division of Critical Care Medicine Seattle Children's Hospital Seattle, Washington

Robert M DiBlasi RRT-NPS FAARC Department of Respiratory Care Seattle Children's Hospital and Research Institute Seattle, Washington

\section{REFERENCES}

1. Krawiec C, Carl D, Stetter C, Kong L, Ceneviva GD, Thomas NJ. Challenges with implementation of a respiratory therapist driven protocol of spontaneous breathing trials in the pediatric intensive care unit. Respir Care 2017;62(10):1233-1240.

2. Ferguson LP, Walsh BK, Munhall D, Arnold JH. A spontaneous breathing trial with pressure support overestimates readiness for extubation in children. Pediatr Crit Care Med 2011;12(6):e330-e335.

3. Rose L, Schultz MJ, Cardwell CR, Jouvet P, McAuley DF, Blackwood B. Automated versus non-automated weaning for reducing the duration of mechanical ventilation for critically ill adults and children. Cochrane Database Syst Rev 2014;(6):CD009235.
4. Jouvet PA, Payen V, Gauvin F, Emeriaud G, Lacroix J. Weaning children from mechanical ventilation with a computer-driven protocol: a pilot trial. Intensive Care Med 2013;39(5):919-25.

5. Ely EW, Baker AM, Dunagan DP, Burke HL, Smith AC, Kelly PT, et al. Effect of the duration of mechanical ventilation of identifying patients capable of breathing spontaneously. N Engl J Med 1996; 335(25):1864-69.

6. Foronda FK, Troster EJ, Farias JA, Barbas CS, Ferraro AA, Faria LS, et al. The impact of daily evaluation and spontaneous breathing test on the duration of pediatric mechanical ventilation: a randomized controlled trial. Crit Care Med 2011;39(11):2526-33.

7. Curley MA, Wypij D, Watson RS, Grant MJ, Asaro LA, Cheiftez IM, et al. Protocolized sedation vs usual care in pediatric patients mechanically ventilated for acute respiratory failure: a randomized clinical trial. JAMA 2015;313(4):379-89.

8. Faustino EV, Gedeit R, Schwarz AJ, Asaro LA, Wypij D, Curley MA, Randomized Evaluation of Sedation Titration for Respiratory Failure (RESTORE) Study Investigators. Accuracy of an extubation readiness test in predicting successful extubation in children with acute respiratory failure from lower respiratory tract disease. Crit Care Med 2017;45(1):94-102.

9. Blackwood B, Burns KE, Cardwell CR, O'Halloran P. Protocolized versus non-protocolized weaning for reducing the duration of invasive mechanical ventilation in critically ill adult patients (review). The Cochrane Library 2014;6(11):CD006904.

10. Mhanna MJ, Anderson IM, Iyer NP, Baumann A. The use of extubation readiness parameters: a survey of pediatric critical care physicians. Respir Care 2014;59(3):334-9.

11. Randolph AG, Wypij D, Venkataraman, Hanson JH, Gedeit RG, Meert KL, et al. Effect of mechanical ventilator weaning protocols on respiratory outcomes in infants and children: a randomized controlled trial. JAMA 2002;288(20):2561-68.

12. Willis BC, Graham AS, Yoon E, Wetzel RC, Newth CJ. Pressurerate products and phase angles in children on minimal support ventilation and after extubation. Intensive Care Med 2005;31(12):1700-5.

13. Khemani RG, Hotz J, Morzov R, Flink RC, Kamerkar A, LaFortune $\mathrm{M}$, et al. Pediatric extubation readiness tests should not use pressure support. Intensive Care Med 2016;42(8):1214-22. 